\title{
Enhancing the Optimization of the Selection of a Product Service System Scheme: A Digital Twin-Driven Framework
}

\author{
Yan $\mathrm{Li}^{1}$ - Lianhui $\mathrm{Li}^{2},{ }^{*}$ \\ ${ }^{1}$ Xinxiang University, Computer and Information Engineering College, China \\ 2 North Minzu University, School of Mechanical and Electrical Engineering, China
}

\begin{abstract}
A product service system (PSS) has been developed for manufacturing enterprises to provide users with personalized products and services. The optimization of PSS scheme selection is a key stage in the PSS design phase. Given the dynamic characteristics of the multi-dimensional influencing factors and their coupling relationships, we propose a digital twin-driven framework to enhance the optimization of PSS scheme selection. The framework is divided into a digital twin layer, an information layer, and an approach layer. The logical relationship between the three layers is given, and a quantitative PSS scheme selection optimization mechanism is designed. Fuzzy numbers and rough boundary intervals are integrated for the attribute value determination of the PSS scheme. A modified TOPSIS developed by replacing Euclidean distance with relational vector distance is adopted for the PSS scheme assessment. A case of an air purification PSS scheme selection optimization under the proposed digital twin driven framework is studied. It is shown that the designed PSS scheme selection optimization mechanism is effective and can be enhanced with the presented framework.
\end{abstract}

Keywords: Product service system, scheme selection, digital twin, fuzzy assessment

Highlights

- The logical relationship of the layers in the digital twin-driven framework can describe the decision-making process of PSS scheme selection optimization more consistently.

- In the designed PSS scheme selection optimization quantitative approach, the assessment opinions of multiple experts are expressed and integrated through fuzzy numbers and rough sets.

- Alternate PSS schemes are assessed by synthesizing attribute importance and modified TOPSIS to achieve PSS scheme selection optimization.

\section{INTRODUCTION}

Service-oriented manufacturing is a new manufacturing mode of integration of the manufacturing service industries. In the context of increasingly fierce market competition, under the service-oriented manufacturing mode, the integration of services and products promotes the value-added of all stakeholders in the manufacturing value chain. Services are no longer attached to products; to a great extent, the service of products determines the value of products in the market and gradually becomes an important factor for enterprises to expand market space and exercise their core competitiveness [1] to [3]. In this context, an integrated solution - a product service system (PSS) - has been developed for manufacturing enterprises to provide users with personalized products and services [4]. Through the integration of tangible products and intangible services, PSS provides enterprises with an overall solution to create high value-added products [5]. However, in the design process of PSS, due to the fuzziness of customer demand and deviations in the understanding of designers regarding customer demand, the PSS scheme is not unique. The quality of the scheme is directly related to customer satisfaction. In order to better meet the personalized needs of customers and improve the market share of enterprises, it is particularly important to assess multiple PSS schemes and achieve PSS scheme selection optimization.

The influencing factors of the optimization of PSS scheme selection have multi-dimensional characteristics and complex coupling relationships. The determination of a PSS scheme needs to go through the stages of design, trial operation, and scheme revision on the time axis. However, the multidimensional factors and their coupling relationship of PSS scheme selection optimization show dynamic characteristics in this process. At present, most of the research is in the form of the static assessment and selection optimization of PSS scheme in the design phase [6] to [9].

Therefore, in the PSS scheme selection optimization, it is necessary to investigate the multidimensional influencing factors and their coupling relationship comprehensively. For the evolution of a PSS scheme on the time axis, the real-time, integrity and logicality of information reflection are urgently required.

A digital twin creates the virtual model of a physical entity in a digital way [10], simulates the behaviour of the physical entity by means of data, and 
has the characteristics of real-time synchronization, faithful mapping and high fidelity through the means of virtual real interaction feedback, data fusion analysis, decision iteration selection optimization [11], so as to promote the interaction and integration of physical world and information world, and increase or expand new capabilities for physical entity [12]. At present, scholars have constructed digital twin frameworks in production control and optimization [10] to [12], which can provide a reference for the establishment of digital twin-driven PSS scheme selection optimization.

Therefore, a digital twin establishes the mapping and interaction between the physical world and the digital world, which can completely and dynamically present the multi-dimensional influencing factors, thus providing a perfect technical guarantee for the PSS scheme selection optimization. After realizing the complete and dynamic mapping interaction between the physical world and digital world in PSS scheme selection optimization based on digital twin, how to comprehensively master the multi-dimensional influencing factors and their coupling relationship for comparative analysis is the key issue.

At present, in the solution of PSS scheme selection optimization, a widely used method is to comprehensively consider the multi-dimensional influencing factors and their coupling relationship that affect PSS scheme determination by using the experience and wisdom of experts. This method is mostly realized by multi-attribute decision-making (MADM) [13] to [15]. Generally, the attribute value is evaluated by a single expert, and the subjective or objective weighting method is used to solve the index weight. The selection optimization of PSS scheme involves multi-dimensional influencing factors with complex coupling relationship, and these factors and their relationship are dynamic evolution. It is difficult for a single expert to comprehend this accurately, and the single weighting method cannot fully reflect the weight information. Therefore, the problem formulation of this paper is described as how to apply the MADM method in which many experts participate to realize the PSS scheme selection optimization on the basis of digital twin driving.

\section{DIGITAL TWIN-DRIVEN PSS SCHEME SELECTION OPTIMIZATION FRAMEWORK AND MECHANISM}

\subsection{Digital twin driven PSS scheme selection optimization framework}

The evolution of a PSS scheme includes redesign, adaptation, and execution. Traditional PSS scheme

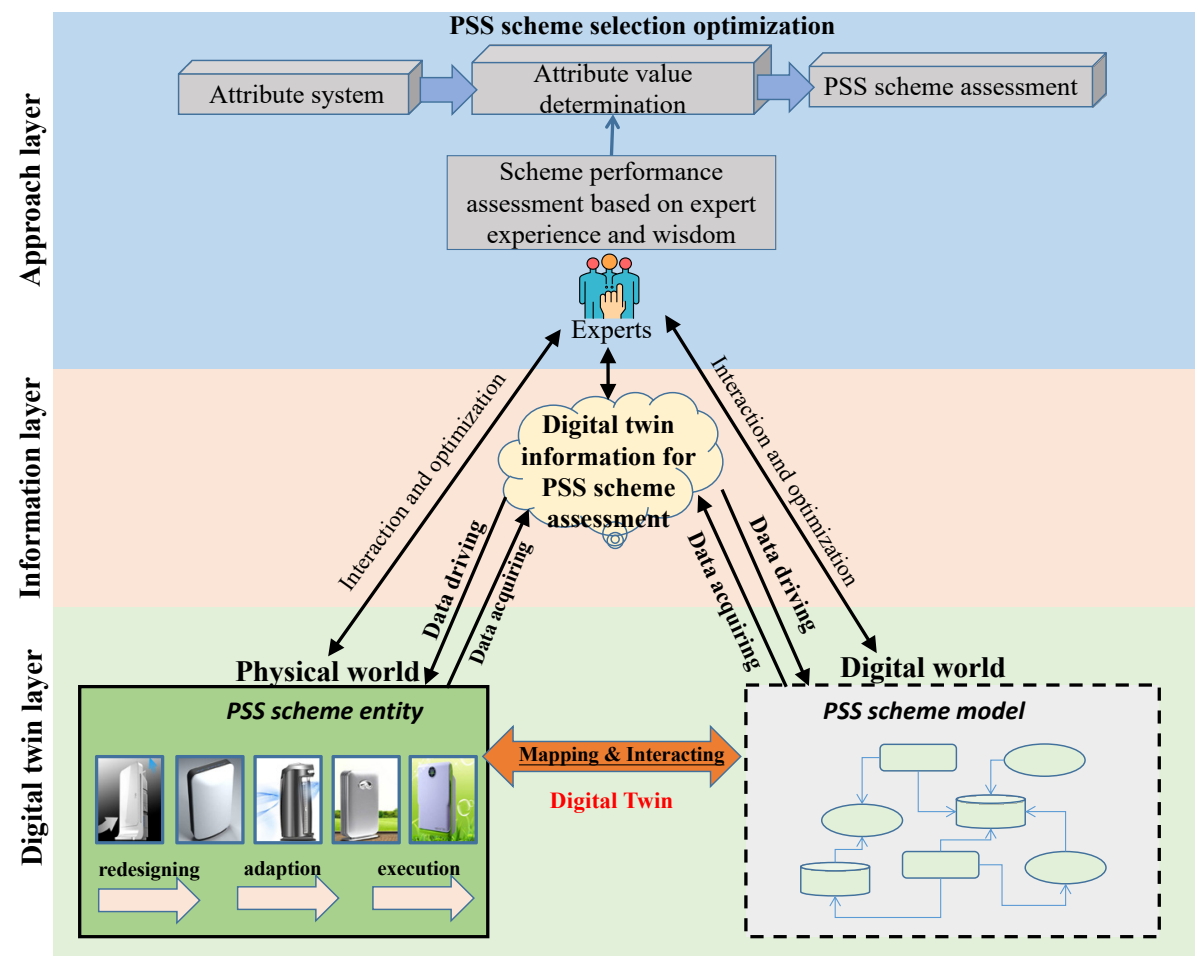

Fig. 1. Digital twin driven PSS scheme selection optimization framework 
selection optimization is usually based on various attributes of the PSS scheme in the redesign phase [5] to [9]. The decision made on this basis is only static and incomplete. However, the attributes of a PSS scheme are also dynamic in the evolution process. Therefore, the traditional PSS scheme selection optimization can not meet the requirements of dynamic and comprehensive decision-making due to the lack of a systematic representation of the dynamic evolution of PSS scheme on the timeline.

Therefore, the implementation of PSS scheme selection optimization needs to build an information fusion framework between the physical system and the virtual system to fully characterize the dynamic evolution of PSS scheme attributes on the timeline in multiple phases. According to the above analysis and the advantages of a digital twin, the digital twin-driven PSS scheme selection optimization framework is proposed, as shown in Fig. 1. Through the bidirectional real mapping and real-time data interaction between entity and model, comprehensive data integration and fusion of PSS scheme entity, PSS scheme model and PSS scheme evaluation approach are realized. Supported by the digital twin information, PSS scheme selection optimization is carried out, and the iterative interaction of selection optimization approach, entity, and model for PSS scheme are realized.
As shown in Fig. 1, the digital twin-driven PSS scheme selection optimization framework is divided into three layers, which are digital twin layer, information layer and approach layer. The logical relationship between the three layers is as follows.

In the digital twin layer, the mapping and interacting between the physical world and the digital world are described theoretically. First, the PSS scheme entity exists in the physical world. In general, the design phase will produce multiple PSS schemes that meet the requirements. Then, in the test running stage, the original PSS scheme will be adjusted and optimized iteratively. Finally, the optimal PSS scheme is determined and put into the formal running stage. The data in the formal running will also be fed back to assist in the selection optimization of scheme design.

Second, the PSS scheme model is a real and complete digital image of the PSS scheme. It integrates all factors related to PSS scheme evaluation and the correlation between them. At the same time, it is a dynamic model that can describe the dynamic evolution of the design, test running and formal running stages of PSS scheme.

In the approach layer, PSS scheme selection optimization approach is driven by digital twin information. Through the interaction with the physical entity and virtual model, the iterative selection

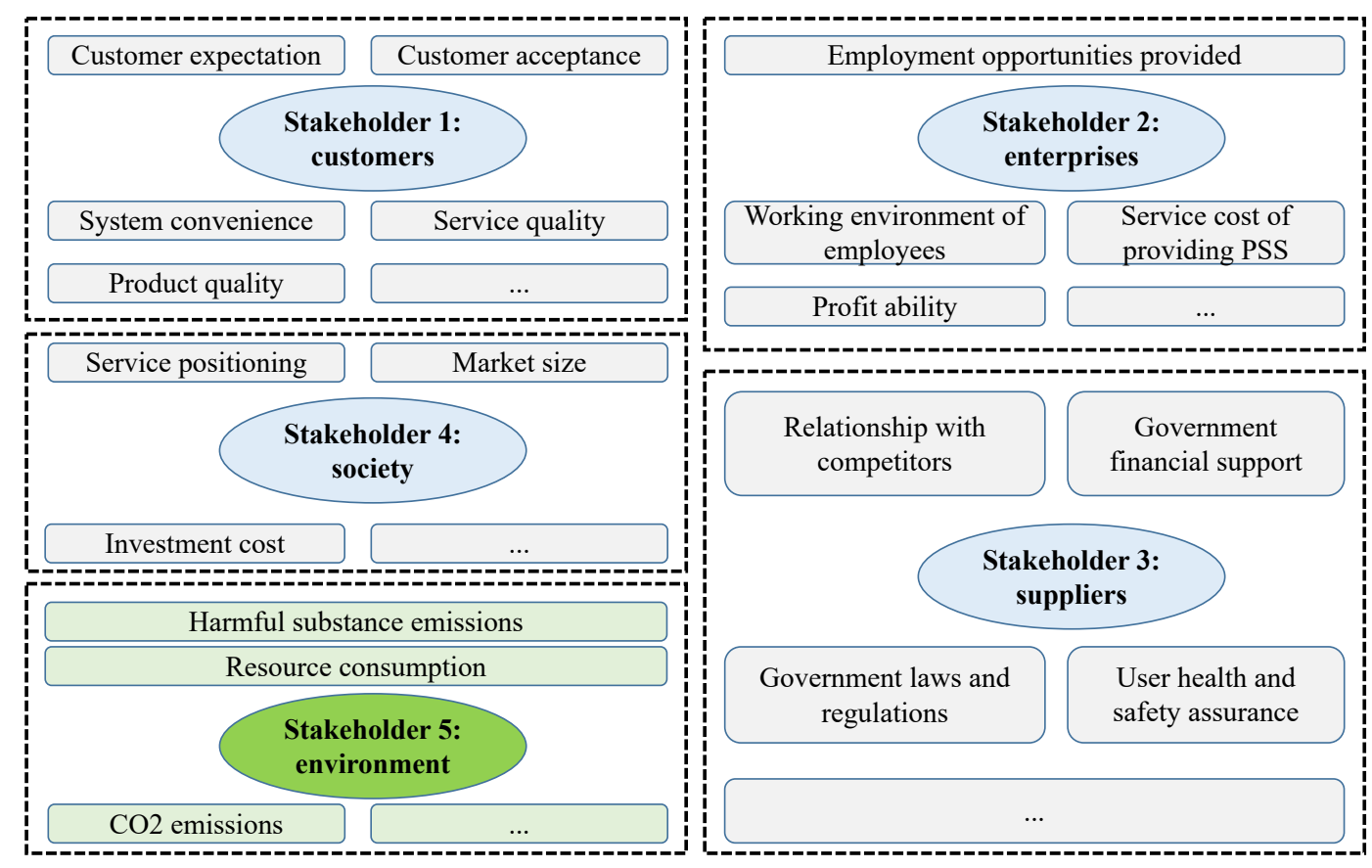

Fig. 2. Attribute system of PSS scheme selection optimization 
optimization is realized with the aid of the intelligent assessment of the PSS scheme.

In the information layer, digital twin information for PSS scheme assessment is the core driver of the PSS scheme selection optimization framework. It mainly includes the initial design data, the virtual model structured data, the real-time data of the running environment, and also includes the new data generated in the running stage.

\subsection{PSS scheme selection optimization mechanism: a quantitative approach}

PSS scheme selection optimization is realized by assessing multiple PSS schemes. At present, the common attribute system for PSS scheme assessment is mainly for some specific industries, with poor universality and less consideration of environmental factors. Therefore, based on the previous research results [1] to [8], this paper adds the environmental attribute of green connotation into the attribute system of PSS scheme assessment. Starting from multiple stakeholders (customers, enterprises, suppliers and society) involved in the PSS scheme design, the influencing factors are summarized, and a macro and comprehensive attribute system of PSS scheme assessment is constructed, without considering the detailed and complicated index factors. The attribute system of PSS scheme selection optimization is composed of multiple stakeholders, and the influencing factors of each interest attribute are shown in Fig. 2.

Based on the attribute system of PSS scheme selection optimization proposed in Fig. 2, a quantitative PSS scheme selection optimization approach is designed, and its detailed steps are as follow.

\subsubsection{Attribute value determination of PSS scheme}

The attribute value determination of PSS scheme selection optimization in the existing research needs to consider multiple qualitative or quantitative factors respectively, and the process of attribute value determination is rather tedious [5] to [9]. In the attribute system constructed in this paper, the attributes of the PSS scheme are all qualitative. The mathematical and statistical characteristics of an expert scoring method can make the best use of expert experience, and the calculation process is very simple. Using expert scoring method for qualitative attribute value determination is a reasonable method.
However, experts' judgment on the relative merits and demerits of multiple PSS schemes on an attribute depends on personal experience and subjective judgment, so it is unreasonable to express them with accurate values. Compared with it, the fuzzy number can reflect the internal uncertainty of experts' judgment. A trapezoid fuzzy number has more complex membership function than triangle fuzzy number [13] and [14]. Using a trapezoid fuzzy number to express expert score value can better describe this uncertainty than triangle fuzzy number.

At the same time, when synthesizing multiple experts' opinions, the experts have obvious ambiguity when judging the attribute value of a PSS scheme. Rough numbers and rough boundary intervals in rough set theory can describe the fuzziness as set boundary regions instead of membership function form, which can better reflect the true judgment of experts and take into account the opinions of multiple experts. Therefore, this paper proposes a fuzzy rough method, which combines fuzzy numbers and rough set theory to calculate the attribute value of the PSS scheme.

Related definitions are as follows.

Definition 1: Rough approximation set. It is assumed that $U$ is a non-empty finite set of objects, which is called domain. $Y$ is any object in $U$. All objects in $U$ belong to $n$ divisions, i.e. $S_{1}, S_{2}, \ldots, S_{n}$. If the $n$ divisions have an order relation $S_{1}<S_{2}<\ldots,<S_{n}$, for any division $S_{w}(1 \leq w \leq n)$, the upper rough approximation set $\overline{A S}\left(S_{w}\right)$ and lower rough approximation set $\underline{A S}\left(S_{w}\right)$ can be defined as:

$$
\begin{aligned}
& \overline{A S}\left(S_{w}\right)=\left\{Y \in K \mid K \subseteq U / R(Y) \wedge K \geq S_{w}\right\}, \\
& \underline{A S}\left(S_{w}\right)=\left\{Y \in K \mid K \subseteq U / R(Y) \wedge K \leq S_{w}\right\},
\end{aligned}
$$

where $U / R(Y)$ indicates the division of fuzzy relation $R$ on $U$.

Definition 2: Rough boundary interval. According to Definition 1, any ambiguous division $S_{i}$ on the domain can be represented by its rough boundary interval. The rough boundary interval is composed of the lower rough limit $\underline{L}\left(S_{w}\right)$ and the upper rough limit $\bar{L}\left(S_{w}\right)$, which are expressed in mathematical form as follows:

$$
\begin{array}{ll}
\underline{L}\left(S_{w}\right)=\frac{\sum R(Y)}{\underline{N}\left(S_{w}\right)}, \quad Y \in \underline{A S}\left(S_{w}\right), \\
\bar{L}\left(S_{w}\right)=\frac{\sum R(Y)}{\bar{N}\left(S_{w}\right)}, \quad Y \in \overline{A S}\left(S_{w}\right),
\end{array}
$$

where $\underline{N}\left(S_{w}\right)$ and $\bar{N}\left(S_{w}\right)$ are the number of objects contained in the lower and upper approximation sets of $S_{w}$, respectively. Therefore, 
the rough boundary interval of $S_{w}$ is represented as $\operatorname{RBI}\left(S_{w}\right)=\left[\underline{L}\left(S_{w}\right), \bar{L}\left(S_{w}\right)\right]$.

The known conditions of attribute value determination of PSS scheme are as follows. There are $l$ alternate PSS schemes and $q$ experts to assess the performance of alternate PSS scheme on each attribute shown in Fig. 2. The attribute set is $A=\left\{A_{1}, A_{2}, \ldots, A_{m}\right\}$ ( $m=5$ ) where $A_{1}, A_{2}, A_{3}, A_{4}, A_{5}$ represent customer interest attribute, enterprise interest attribute, supplier interest attribute, society interest attribute and environment interest attribute successively.

The detailed process of attribute value determination is as follows.

Step 1. For attribute $A_{t} \in A \quad(t=1,2, \ldots, 5)$, the fuzzy reciprocal assessment matrix given by expert $k$ $(k=1,2, \ldots, q)$ is as follows:

$$
\tilde{E}^{k, t}=\left[\tilde{e}_{i, j}^{k, t}\right]_{l \times l},
$$

where $\tilde{e}_{i, j}^{k, t}$ is the score of alternate PSS scheme $i$ relative to alternate PSS scheme $j$ given by expert $k$ on attribute $A_{t} . \tilde{e}_{i, j}^{k, t}$ is a trapezoid fuzzy number and $\tilde{e}_{i, j}^{k, t}=\left(\alpha_{i, j}^{k, t}, \beta_{i, j}^{k, t}, \gamma_{i, j}^{k, t}, \theta_{i, j}^{k, t}\right)$. If $i=j, \tilde{e}_{i, j}^{k, t}=(1,1,1,1)$. The next step can only be carried out after $q$ assessment matrices are qualified in consistency inspection. Otherwise, the corresponding expert will adjust the assessment matrix.

Step 2. The group assessment matrix is constructed as follows.

$$
\tilde{E}^{t}=\left[\tilde{e}_{i, j}^{t}\right]_{l \times l},
$$

where $\tilde{e}_{i, j}^{t}=\left\{\tilde{e}_{i, j}^{1, t}, \tilde{e}_{i, j}^{2, t}, \ldots, \tilde{e}_{i, j}^{q, t}\right\}$.

Step 3. According to Definitions 1 and 2, the rough boundary interval of $\tilde{e}_{i, j}^{k, t}$ in $\tilde{e}_{i, j}^{t}=\left\{\tilde{e}_{i, j}^{1, t}, \tilde{e}_{i, j}^{2, t}, \ldots, \tilde{e}_{i, j}^{q, t}\right\}$ can be expressed as follows.

$$
R B I\left(\tilde{e}_{i, j}^{k, t}\right)=\left[\underline{L}\left(\tilde{e}_{i, j}^{k, t}\right), \bar{L}\left(\tilde{e}_{i, j}^{k, t}\right)\right],
$$

where $\underline{L}\left(\tilde{e}_{i, j}^{k, t}\right)=\sum_{\tilde{e}_{i, j}^{k, t} \leq \tilde{e}_{i, j}^{k, t}} \tilde{e}_{i, j}^{k, t} / \underline{N}\left(\tilde{e}_{i, j}^{k, t}\right)$ and $\bar{L}\left(\tilde{e}_{i, j}^{k, t}\right)=\sum_{\tilde{e}_{i, j}^{k, t} \geq \tilde{e}_{i, j}^{k, t}} \tilde{e}_{i, j}^{\bar{k}, t} / \bar{N}\left(\tilde{e}_{i, j}^{k, t}\right)$.

Therefore, the rough boundary interval of $\tilde{e}_{i, j}^{t}$ can be expressed as follows.

$\operatorname{RBI}\left(\tilde{e}_{i, j}^{t}\right)=\left[\underline{L}\left(\tilde{e}_{i, j}^{t}\right), \bar{L}\left(\tilde{e}_{i, j}^{t}\right)\right]=\left[\frac{\sum_{k=1}^{q} \underline{L}\left(\tilde{e}_{i, j}^{k, t}\right)}{q}, \frac{\sum_{k=1}^{q} \bar{L}\left(\tilde{e}_{i, j}^{k, t}\right)}{q}\right] .(6)$
Step 4. The rough boundary interval assessment matrix is constructed as $\tilde{\Theta}^{t}=\left[R B I\left(\tilde{e}_{i, j}^{t}\right)\right]_{l \times l}$. Then, $\tilde{\Theta}^{t}$ is split into two matrices as follows.

$$
\begin{aligned}
& \tilde{\varphi}^{t}=\left[\underline{L}\left(\tilde{e}_{i, j}^{t}\right)\right]_{l \times l}, \\
& \tilde{\eta}^{t}=\left[\bar{L}\left(\tilde{e}_{i, j}^{t}\right)\right]_{l \times l},
\end{aligned}
$$

where $\tilde{\varphi}^{t}$ is the rough lower boundary matrix and $\tilde{\eta}^{t}$ is the rough upper boundary matrix.

According to the gravity centre principle of triangular fuzzy number, $\tilde{\varphi}^{t}$ and $\tilde{\eta}^{t}$ are mapped into $\varphi^{t}$ and $\eta^{t}$ respectively, which are in the real number form. Then the eigenvectors of $\varphi^{t}$ and $\eta^{t}$ corresponding to the maximum eigenvalue are obtained, respectively, as follows.

$$
\begin{aligned}
& z\left(\varphi^{t}\right)=\left[z_{1}\left(\varphi^{t}\right), z_{2}\left(\varphi^{t}\right), \ldots, z_{l}\left(\varphi^{t}\right)\right], \\
& z\left(\eta^{t}\right)=\left[z_{1}\left(\eta^{t}\right), z_{2}\left(\eta^{t}\right), \ldots, z_{l}\left(\eta^{t}\right)\right] .
\end{aligned}
$$

Step 5. After averaging the two eigenvectors obtained in Step 5, we can get a vector as follows.

$$
z^{t}=\left[z_{1, t}, z_{2, t}, \ldots, z_{l, t}\right] .
$$

where $z_{i, t}=\left(z_{i}\left(\varphi^{t}\right)+z_{i}\left(\eta^{t}\right)\right) / 2$ is the attribute value of alternate PSS scheme $i$ on attribute $A_{t}$.

The attribute values of $l$ alternate PSS schemes on other attributes can be obtained by a similar way. At last, the attribute value matrix is obtained as $Z=\left[z_{i, t}\right]_{l \times m}$.

\subsubsection{PSS Scheme Assessment Based on Modified TOPSIS}

Based on attribute value matrix $Z=\left[z_{i, t}\right]_{l \times m}$, multiple types of attribute importance can be obtained by different weighting methods, such as entropy weight method, standard deviation method and CRITIC. Each type of attribute importance contains valid information for PSS scheme assessment. Therefore, we synthesize multiple types of attribute importance. It is assumed that the attribute importance has $p$ types. The attribute importance vectors are $\omega^{1}=\left[\omega_{1}^{1}, \omega_{2}^{1}, \ldots, \omega_{m}^{1}\right]$, $\omega^{2}=\left[\omega_{1}^{2}, \omega_{2}^{2}, \ldots, \omega_{m}^{2}\right], \ldots, \omega^{p}=\left[\omega_{1}^{p}, \omega_{2}^{p}, \ldots, \omega_{m}^{p}\right]$. The synthesized attribute importance vector is as follows.

$$
\omega=\left[\omega_{1}, \omega_{2}, \ldots, \omega_{m}\right],
$$

where $\omega_{t}=\sum_{h=1}^{p} \omega_{t}^{h} / p$. Then the attribute value matrix with importance information is obtained as $Y=\left[y_{i, t}\right]_{l \times m}$, here $y_{i, t}=\omega_{t} z_{i, t}$. 
Next, we propose a modified TOPSIS by replacing Euclidean distance with relational vector distance based on set pair analysis theory. In traditional TOPSIS [16] and [17], the Euclidean distance from assessment object to the ideal solution is adopted for closeness calculation. However, the objects on the perpendicular bisector of two ideal solutions have the same closeness and cannot be distinguished. The principles of using proposed modified TOPSIS to realize the PSS scheme assessment are as follows.

(1) An assessment object (i.e., a PSS scheme) and two ideal solutions (i.e., positive and negative ideal solutions) constitute two set pairs, respectively.

(2) The set pair is then decomposed into multiple element pairs.

(3) For each element pair, the sameness, contrariety and difference relationships are analysed to obtain the relationship between assessment object and ideal solution. Then the relational vector distance from the assessment object to the ideal solution is obtained.

(4) According to the arranging rule of TOPSIS, the closeness of assessment object is calculated based on its relational vector distances. Finally, all assessment objects are arranged according to their closeness values.

The detailed process of PSS scheme assessment based on modified TOPSIS is as follows.

$\underline{\text { Step } 1}$. Set pairs formed by assessment object and two ideal solutions are constructed.

Based on $Y=\left[y_{i, t}\right]_{l \times m}$, the positive ideal solution is obtained as $Y^{+}=\left[y_{1}^{+}, y_{2}^{+}, \ldots, y_{m}^{+}\right]$and the negative ideal solution is obtained as $Y^{-}=\left[y_{1}^{-}, y_{2}^{-}, \ldots, y_{m}^{-}\right]$, where $y_{t}^{+}=\max \left\{y_{1, t}, y_{2, t}, \ldots, y_{l, t}\right\} \quad$ and $y_{t}^{-}=\min \left\{y_{1, t}, y_{2, t}, \ldots, y_{l, t}\right\} . \quad Y_{i}=\left[y_{i, 1}, y_{i, 2}, \ldots, y_{i, m}\right]$ represents PSS scheme $i$. Based on set pair analysis theory [18] to [20], $Y_{i}$ and $Y^{+}$form a set pair, which is expressed by $<Y_{i}, Y^{+}>$, while $Y_{i}$ and $Y^{-}$form a set pair which is expressed by $\left\langle Y_{i}, Y^{>}>\right.$.

Through comparing the values, $S_{i}^{+}$element pairs have tiny difference (sameness relationship), $C_{i}^{+}$element pairs have huge difference (contrariety relationship) and $D_{i}^{+}$element pairs have not very obvious difference (difference relationship). Here, $S_{i}^{+}+C_{i}^{+}+D_{i}^{+}=m$. Therefore, the relational degree of set pair $\left\langle Y_{i}, Y^{+}\right\rangle$, which characterizes the uncertain quantitative relationship between $Y_{i}$ and $Y^{+}$, is expressed as follows:

$$
\rho_{i}^{+}=s_{i}^{+} \Delta^{\prime}+c_{i}^{+} \Delta^{\prime \prime}+d_{i}^{+} \Delta^{\prime \prime \prime},
$$

where $\Delta^{\prime}, \Delta^{\prime \prime}, \Delta^{\prime \prime \prime}$ indicate the sameness, contrariety and difference relationships respectively, and $s_{i}^{+}=S_{i}^{+} / m, c_{i}^{+}=C_{i}^{+} / m$ and $d_{i}^{+}=D_{i}^{+} / m$ indicate the sameness, contrariety and difference coefficients, respectively.

The relational vector of set pair $\left\langle Y_{i}, Y^{+}\right\rangle$is as follows:

$$
\mu_{i}^{+}=\left[s_{i}^{+}, c_{i}^{+}, d_{i}^{+}\right] .
$$

Similarly, the relational degree of set pair $\left\langle Y_{i}, Y^{>}\right\rangle$ is expressed as follows:

$$
\rho_{i}^{-}=s_{i}^{-} \Delta^{\prime}+c_{i}^{-} \Delta^{\prime \prime}+d_{i}^{-} \Delta^{\prime \prime \prime} .
$$

The relational vector of set pair $\left\langle Y_{i}, Y^{-}\right\rangle$is as follows:

$$
\mu_{i}^{-}=\left[s_{i}^{-}, c_{i}^{-}, d_{i}^{-}\right]
$$

Step 2. Set pair is divided into several element pairs.

Set pair $\left.<Y_{i}, Y^{+}\right\rangle$consists of $m$ element pairs $<y_{i, 1}, y_{1}^{+}>,<y_{i, 1}, y_{2}^{+}>, \ldots,<y_{i, m}, y_{m}^{+}>$. For element pair $\left\langle y_{i, t}, y_{t}^{+}\right\rangle$, its relational degree can be expressed as follows:

$$
\rho_{i, t}^{+}=s_{i, t}^{+} \Delta^{\prime}+c_{i, t}^{+} \Delta^{\prime \prime}+d_{i, t}^{+} \Delta^{\prime \prime \prime},
$$

where $\Delta^{\prime}, \Delta^{\prime \prime}, \Delta^{\prime \prime \prime}$ indicate the sameness, contrariety and difference relationships respectively, and $s_{i, t}^{+}$, $c_{i, t}^{+}$and $d_{i, t}^{+}$indicate the sameness, contrariety and difference coefficients of element pair $\left\langle y_{i, t}, y_{t}^{+}\right\rangle$ respectively. If $y_{i, t}=y_{t}^{+}$, then $s_{i, t}^{+}=1, \quad c_{i, t}^{+}=0$ and $d_{i, t}^{+}=0$. If $y_{i, t}=y_{t}^{-}$, then $s_{i, t}^{+}=0, \quad c_{i, t}^{+}=1$ and $d_{i, t}^{+}=0$. If $y_{t}^{-}<y_{i, t}<y_{t}^{+}$, then $s_{i, t}^{+}=0, \quad c_{i, t}^{+}=0$ and $d_{i, t}^{+}=\left(y_{t}^{+}-y_{i, t}\right) /\left(y_{t}^{+}-y_{t}^{-}\right)$. Therefore, the relational degree of $<y_{i, t}, y_{t}^{+}>$is:

$$
\rho_{i,}^{+}=\frac{\sum_{t=1}^{m} s_{i, t}^{+}}{m} \Delta^{\prime}+\frac{\sum_{t=1}^{m} c_{i, t}^{+}}{m} \Delta^{\prime \prime}+\frac{\sum_{t=1}^{m} d_{i, t}^{+}}{m} \Delta^{\prime \prime \prime} .
$$

Similarly, for set pair $\left\langle Y_{i}, Y^{>}\right\rangle$formed by $Y_{i}$ and $Y^{-}$, it consists of $m$ element pairs $<y_{i, 1}, y_{1}^{-}>,<y_{i, 2}, y_{2}^{-}>, \ldots,<y_{i, m}, y_{m}^{-}>$. The relational degree of element pair $\left\langle y_{i, t}, y_{t}^{-}\right\rangle$is expressed as follows:

$$
\rho_{i, t}^{-}=s_{i, t}^{-} \Delta^{\prime}+c_{i, t}^{-} \Delta^{\prime \prime}+d_{i, t}^{-} \Delta^{\prime \prime \prime},
$$

where $s_{i, t}^{-}, c_{i, t}^{-}$and $d_{i, t}^{-}$indicate the sameness, contrariety and difference coefficients of element pair $<y_{i, t}, y_{t}^{-}>$respectively. If $y_{i, t}=y_{t}^{+}$, then, then $s_{i, t}^{-}$ $=0, c_{i, t}^{-}=1$ and $d_{i, t}^{-}=0$. If $y_{i, t}=y_{t}^{-}$, then $s_{i, t}^{-}=1, c_{i, t}^{-}=0$ and $d_{i, t}^{-}=0$. If $y_{t}^{-}<y_{i, t}<y_{t}^{+}$, then $s_{i, t}^{+}=0, c_{i, t}^{+}=0$ and 
$d_{i, t}^{-}=\left(y_{i, t}-y_{t}^{-}\right) /\left(y_{t}^{+}-y_{t}^{-}\right)$. Therefore, the relational degree of $\left\langle y_{i, t}, y_{t}^{-}>\right.$is:

$$
\rho_{i}^{-}=\frac{\sum_{t=1}^{m} s_{i, t}^{-}}{m} \Delta^{\prime}+\frac{\sum_{t=1}^{m} c_{i, t}^{-}}{m} \Delta^{\prime \prime}+\frac{\sum_{t=1}^{m} d_{i, t}^{-}}{m} \Delta^{\prime \prime \prime} .
$$

$\underline{\text { Step } 3}$. Set pair formed by assessment object and itself is constructed.

$Y_{i}$ and $Y_{i}$ can form a set pair $\left\langle Y_{i}, Y_{i}\right\rangle$. Because a set is the same as itself, the relational degree of set pair $\left\langle Y_{i}, Y_{i}\right\rangle$ is $\rho_{i}^{i}=1 \cdot \Delta^{\prime}+0 \cdot \Delta^{\prime \prime}+0 \cdot \Delta^{\prime \prime \prime}$. Therefore, its relational vector is $\mu_{i}^{i}=[1,0,0]$.

Step 4. The relational vector distance from the assessment object to the ideal solution is calculated.

Because the relational vector of set pairs $\left\langle Y_{i}, Y^{+}\right\rangle$ and $\left\langle Y_{i}, Y_{i}>\right.$ are $\mu_{i}^{+}=\left[s_{i}^{+}, c_{i}^{+}, d_{i}^{+}\right]$and $\mu_{i}^{i}=[1,0,0]$, respectively, the relational vector distance from assessment object $Y_{i}$ to ideal solution $Y^{+}$is calculated as follows.

$$
\tau_{i}^{+}=\sqrt{\left(s_{i}^{+}-1\right)^{2}+\left(c_{i}^{+}\right)^{2}+\left(d_{i}^{+}\right)^{2}} .
$$

Similarly, the relational vector distance from assessment object $Y_{i}$ to ideal solution $Y^{-}$is calculated as follows.

$$
\tau_{i}^{-}=\sqrt{\left(s_{i}^{-}-1\right)^{2}+\left(c_{i}^{-}\right)^{2}+\left(d_{i}^{-}\right)^{2}} .
$$

Step 5. The closeness of assessment object to positive ideal solution is calculated and adopted as the arranging basis.

By replacing the Euclidean distance with the relational vector distance, TOPSIS is modified. For $Y_{i}$, its closeness to the positive ideal solution is calculated as follows.

$$
\lambda_{i}=\frac{\tau_{i}^{-}}{\tau_{i}^{+}+\tau_{i}^{-}} .
$$

According to the arranging rule of TOPSIS, all assessment objects are arranged according to their closeness values. The PSS scheme with the biggest closeness value is the best one, and the PSS scheme selection optimization is achieved.

\section{CASE STUDY}

In order to enhance the competitiveness of the enterprise, to meet the growing personalized needs of consumers, and to improve the efficiency of product sales, an air purifier manufacturing enterprise began to plan and build an air purification PSS (AirP-PSS). With the AirP-PSS, the enterprise sells the promised level of air purification effectiveness instead of selling air purifiers. In the design phase, several AirP-PSS schemes with different characteristics are provided by PSS design engineers. To achieve AirP-PSS scheme selection optimization, the enterprise will carry out AirP-PSS scheme assessment and determine the best one. There are five alternate AirP-PSS schemes: intelligent series $\left(P_{1}\right)$, energy saving series $\left(P_{2}\right)$, portable series $\left(P_{3}\right)$, sterilization series $\left(P_{4}\right)$ and humidification series $\left(P_{5}\right)$.

Based on the membership function of trapezoid fuzzy number, natural number $N$ can be converted to trapezoid fuzzy number $\tilde{N}$ as follows.

$$
\tilde{N}= \begin{cases}\left(1,1, \frac{3}{2}, 2\right), & N=1 \\ \left(N-1, \frac{N-1}{2}, \frac{N+1}{2}, N+1\right), & 1<N<9 . \\ \left(8, \frac{17}{2}, 9,9\right), & N=9\end{cases}
$$

According to the arithmetic rules of the trapezoid fuzzy number, the commonly used nine-level scale assessment comments and values are fuzzified to get the corresponding trapezoid fuzzy number, as shown in Table 1.

Table 1. Comments and fuzzifieded values of nine-level scale assessment

\begin{tabular}{lc}
\hline \multicolumn{1}{c}{ Comment } & Fuzzed value \\
\hline Extremely superior & $\tilde{9} / \tilde{1}=(4,17 / 3,9,9)$ \\
\hline Strongly superior & $\tilde{8} / \tilde{2}=(7 / 3,3,17 / 3,9)$ \\
\hline Obviously superior & $\tilde{7} / \tilde{3}=(3 / 2,13 / 7,3,4)$ \\
\hline Slightly superior & $\tilde{6} / \tilde{4}=(1,11 / 9,13 / 7,7 / 3)$ \\
\hline Equal & $\tilde{5} / \tilde{5}=(1,1,1,1)$ \\
\hline Slightly inferior & $\tilde{4} / \tilde{6}=(3 / 7,7 / 13,9 / 11,1)$ \\
\hline Obviously inferior & $\tilde{3} / \tilde{7}=(1 / 4,1 / 3,7 / 13,2 / 3)$ \\
\hline Strongly inferior & $\tilde{2} / \tilde{8}=(1 / 9,3 / 17,1 / 3,3 / 7)$ \\
\hline Extremely inferior & $\tilde{1} / \tilde{9}=(1 / 9,1 / 9,3 / 17,1 / 4)$ \\
\hline
\end{tabular}

Based on the digital twin-driven PSS scheme selection optimization framework shown in Fig. 1, the AirP-PSS scheme assessment is done after the experts master the digital twin information comprehensively. The detailed process is as follows.

Three experts participate in the AirP-PSS scheme assessment. For attribute $A_{1}$, the fuzzy reciprocal assessment matrices given by three experts are $E^{1,1}$, $E^{2,1}$ and $E^{3,1}$ as shown in Tables 2, 3, and 4. 
Table 2. Fuzzy reciprocal assessment $E^{1,1}$

\begin{tabular}{lccccc}
\hline & $P_{1}$ & $P_{2}$ & $P_{3}$ & $P_{4}$ & $P_{5}$ \\
\hline$P_{1}$ & $\tilde{5} / \tilde{5}$ & $\tilde{5} / \tilde{5}$ & $\tilde{6} / \tilde{4}$ & $\tilde{5} / \tilde{5}$ & $\tilde{6} / \tilde{4}$ \\
\hline$P_{2}$ & $\tilde{5} / \tilde{5}$ & $\tilde{5} / \tilde{5}$ & $\tilde{6} / \tilde{4}$ & $\tilde{5} / \tilde{5}$ & $\tilde{6} / \tilde{4}$ \\
\hline$P_{3}$ & $\tilde{4} / \tilde{6}$ & $\tilde{4} / \tilde{6}$ & $\tilde{5} / \tilde{5}$ & $\tilde{4} / \tilde{6}$ & $\tilde{5} / \tilde{5}$ \\
\hline$P_{4}$ & $\tilde{5} / \tilde{5}$ & $\tilde{5} / \tilde{5}$ & $\tilde{6} / \tilde{4}$ & $\tilde{5} / \tilde{5}$ & $\tilde{6} / \tilde{4}$ \\
\hline$P_{5}$ & $\tilde{4} / \tilde{6}$ & $\tilde{4} / \tilde{6}$ & $\tilde{5} / \tilde{5}$ & $\tilde{4} / \tilde{6}$ & $\tilde{5} / \tilde{5}$ \\
\hline
\end{tabular}

Table 3. Fuzzy reciprocal assessment $E^{2,1}$

\begin{tabular}{lccccc}
\hline & $P_{1}$ & $P_{2}$ & $P_{3}$ & $P_{4}$ & $P_{5}$ \\
\hline$P_{1}$ & $\tilde{5} / \tilde{5}$ & $\tilde{7} / \tilde{3}$ & $\tilde{5} / \tilde{5}$ & $\tilde{6} / \tilde{4}$ & $\tilde{7} / \tilde{3}$ \\
\hline$P_{2}$ & $\tilde{3} / \tilde{7}$ & $\tilde{5} / \tilde{5}$ & $\tilde{3} / \tilde{7}$ & $\tilde{4} / \tilde{6}$ & $\tilde{5} / \tilde{5}$ \\
\hline$P_{3}$ & $\tilde{5} / \tilde{5}$ & $\tilde{7} / \tilde{3}$ & $\tilde{5} / \tilde{5}$ & $\tilde{6} / \tilde{4}$ & $\tilde{7} / \tilde{3}$ \\
\hline$P_{4}$ & $\tilde{4} / \tilde{6}$ & $\tilde{6} / \tilde{4}$ & $\tilde{4} / \tilde{6}$ & $\tilde{5} / \tilde{5}$ & $\tilde{6} / \tilde{4}$ \\
\hline$P_{5}$ & $\tilde{3} / \tilde{7}$ & $\tilde{5} / \tilde{5}$ & $\tilde{3} / \tilde{7}$ & $\tilde{4} / \tilde{6}$ & $\tilde{5} / \tilde{5}$ \\
\hline
\end{tabular}

Table 4. Fuzzy reciprocal assessment $E^{3,1}$

\begin{tabular}{lccccc}
\hline & $P_{1}$ & $P_{2}$ & $P_{3}$ & $P_{4}$ & $P_{5}$ \\
\hline$P_{1}$ & $\tilde{5} / \tilde{5}$ & $\tilde{6} / \tilde{4}$ & $\tilde{6} / \tilde{4}$ & $\tilde{7} / \tilde{3}$ & $\tilde{6} / \tilde{4}$ \\
\hline$P_{2}$ & $\tilde{4} / \tilde{6}$ & $\tilde{5} / \tilde{5}$ & $\tilde{6} / \tilde{4}$ & $\tilde{6} / \tilde{4}$ & $\tilde{5} / \tilde{5}$ \\
\hline$P_{3}$ & $\tilde{4} / \tilde{6}$ & $\tilde{4} / \tilde{6}$ & $\tilde{5} / \tilde{5}$ & $\tilde{6} / \tilde{4}$ & $\tilde{4} / \tilde{6}$ \\
\hline$P_{4}$ & $\tilde{3} / \tilde{7}$ & $\tilde{4} / \tilde{6}$ & $\tilde{4} / \tilde{6}$ & $\tilde{5} / \tilde{5}$ & $\tilde{4} / \tilde{6}$ \\
\hline$P_{5}$ & $\tilde{4} / \tilde{6}$ & $\tilde{5} / \tilde{5}$ & $\tilde{6} / \tilde{4}$ & $\tilde{6} / \tilde{4}$ & $\tilde{5} / \tilde{5}$ \\
\hline
\end{tabular}

$E^{1,1}, E^{2,1}$ and $E^{3,1}$ are all qualified by consistency inspection. The group assessment matrix is constructed as $\tilde{E}^{1}=\left[\tilde{e}_{i, j}^{1}\right]_{5 \times 5}$ in which $\tilde{e}_{i, j}^{1}=\left\{\tilde{e}_{i, j}^{1,1}, \tilde{e}_{i, j}^{2,1}, \tilde{e}_{i, j}^{3,1}\right\}$.
According to Eqs. (5) and (6), the rough boundary interval of $\tilde{e}_{1,2}^{1}$ can be obtained as: $\operatorname{RBI}\left(\tilde{e}_{1,2}^{1}\right)=[(1.0556$, $1.1570,1.4603,1.7037),(1.3056,1.5855,2.4603$, 3.2037)].

After calculating the rough boundary intervals of other elements in $\tilde{E}^{1}=\left[\tilde{e}_{i, j}^{1}\right]_{5 \times 5}$, the rough boundary interval assessment matrix is constructed as $\tilde{\Theta}^{1}=\left[R B I\left(\tilde{e}_{i, j}^{1}\right)\right]_{5 \times 5}$. Then, $\tilde{\Theta}^{t}$ is split into rough lower boundary matrix $\tilde{\varphi}^{1}=\left[\underline{L}\left(\tilde{e}_{i, j}^{1}\right)\right]_{5 \times 5}$ and rough upper boundary matrix $\tilde{\eta}^{1}=\left[\bar{L}\left(\tilde{e}_{i, j}^{1}\right)\right]_{5 \times 5}$ as shown in Tables 5 and 6.

According to the gravity centre principle of triangular fuzzy number, $\tilde{\varphi}^{1}$ and $\tilde{\eta}^{1}$ are mapped into the real number form $\tilde{\varphi}^{1}$ and $\tilde{\eta}^{1}$ respectively. Then the eigenvectors of $\tilde{\varphi}^{1}$ and $\tilde{\eta}^{1}$ corresponding to the maximum eigenvalue are obtained as

$z\left(\varphi^{1}\right)=[-0.6066,-0.4054,-0.4335,-0.3925,-0.3545]$ and

$z\left(\eta^{1}\right)=[-0.5899,-0.4043,-0.4621,-0.3940,-0.3462]$, respectively.

After averaging the two eigenvectors, the attribute value vector of five alternate AirP-PSS schemes on attribute $A_{1}$ is obtained as $z^{1}=[0.5983,0.4048,0.4478$, $0.3933,0.3504]$. Finally, we obtain other attribute values of five alternate AirP-PSS schemes. The attribute value matrix $Z=\left[z_{i, t}\right]_{5 \times 5}$ is shown in Table 7.

Based on attribute value matrix $Z=\left[z_{i, t}\right]_{l \times m}$, the attribute importance vectors are obtained as: $\omega^{1}=[0.2089,0.2007,0.1997,0.1819,0.2088]$, $\omega^{2}=[0.1111,0.2469,0.1887,0.3244,0.1289]$ and $\omega^{2}=[0.1962,0.2049,0.1778,0.2575,0.1637]$, which are by entropy weight method, standard deviation method and CRITIC method, respectively.

Table 5. Rough lower boundary matrix $\tilde{\varphi}^{1}=\left[\underline{L}\left(\tilde{e}_{i, j}^{1}\right)\right]_{5 \times 5}$

$$
\begin{array}{rrrrr}
P_{1} & P_{2} & P_{3} & P_{4} & P_{5}
\end{array}
$$

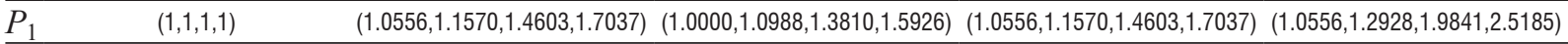

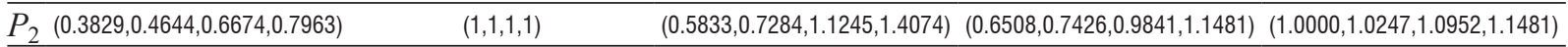
\begin{tabular}{lllll}
\hline$P_{3}(0.4921,0.5897,0.8384,1.0000)$ & $(0.5476,0.6850,1.0606,1.3333)$ & $(1,1,1,1)$ & $(0.6825,0.8424,1.2799,1.5926)$ & $(0.7063,0.8132,1.1111,1.3333)$
\end{tabular} \begin{tabular}{llllll}
\hline$P_{4}(0.3829,0.4644,0.6674,0.7963)$ & $(0.6508,0.7426,0.9841,1.1481)$ & $(0.4921,0.6144,0.9336,1.1481)$ & $(1,1,1,1)$ & $(0.6825,0.8424,1.2799,1.5926)$ \\
\hline
\end{tabular}

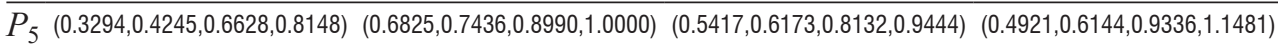
$(1,1,1,1)$

Table 6. Rough upper boundary matrix $\tilde{\eta}^{1}=\left[\bar{L}\left(\tilde{e}_{i, j}^{1}\right)\right]_{5 \times 5}$

\begin{tabular}{|c|c|c|c|c|}
\hline$P_{1}$ & $P_{2}$ & $P_{3}$ & $P_{4}$ & $P_{5}$ \\
\hline$(1,1,1,1)$ & $(1.3056,1.5855,2.4603,3.2037)$ & $(1.0000,1.1975,1.7619,2.1852)$ & $(1.3056,1.5855,2.4603,3.2037)$ & $(1.2778,1.5750,2.4921,3.2593)$ \\
\hline$P_{2}(0.7579,0.7977,0.8982,0.9630)$ & $(1,1,1,1)$ & $(0.9167,1.1235,1.7106,2.1481)$ & $(0.9365,1.0845,1.5036,1.8148)$ & $(1.0000,1.1235,1.4762,1.7407)$ \\
\hline$P_{3}(0.7460,0.7949,0.9192,1.0000)$ & $(1.0238,1.2711,2.0303,2.6667)$ & $(1,1,1,1)$ & $(0.9365,1.1462,1.7417,2.1852)$ & $(1.2421,1.4725,2.2020,2.8333)$ \\
\hline$P_{4}(0.7579,0.7977,0.8982,0.9630)$ & $(0.9365,1.0845,1.5036,1.8148)$ & $(0.7460,0.9183,1.3954,1.7407)$ & $(1,1,1,1)$ & $(0.9365,1.1462,1.7417,2.1852)$ \\
\hline$P_{5}(0.4087,0.5157,0.7871,0.9630)$ & $(0.9365,0.9487,0.9798,1.0000)$ & $(0.9167,1.0617,1.4725,1.7778)$ & $(0.7460,0.9183,1.3954,1.7407)$ & $(1,1,1,1)$ \\
\hline
\end{tabular}


Table 7. Attribute value matrix $Z=\left[z_{i, t}\right]_{5 \times 5}$

\begin{tabular}{lccccc}
\hline & $A_{1}$ & $A_{2}$ & $A_{3}$ & $A_{4}$ & $A_{5}$ \\
\hline$P_{1}$ & 0.5983 & 0.2270 & 0.3785 & 0.3419 & 0.5575 \\
\hline$P_{2}$ & 0.4048 & 0.5469 & 0.5706 & 0.1576 & 0.6324 \\
\hline$P_{3}$ & 0.4478 & 0.6572 & 0.4218 & 0.0975 & 0.4649 \\
\hline$P_{4}$ & 0.3933 & 0.3157 & 0.2357 & 0.7922 & 0.3595 \\
\hline$P_{5}$ & 0.3504 & 0.7134 & 0.1557 & 0.4854 & 0.6003 \\
\hline
\end{tabular}

Then, the synthesized attribute importance vector is obtained as $\omega=[0.1721,0.2175,0.1887,0.2546$, $0.1671]$. The attribute value matrix with importance information is obtained as $Y=\left[y_{i, t}\right]_{5 \times 5}$, as shown in Table 8, where $y_{i, t}=\omega_{t} z_{i, t}$.

Table 8. Attribute value matrix with importance information $Y=\left[y_{i, t}\right]_{5 \times 5}$

\begin{tabular}{lccccc}
\hline & $A_{1}$ & $A_{2}$ & $A_{3}$ & $A_{4}$ & $A_{5}$ \\
\hline$P_{1}$ & 0.1030 & 0.0494 & 0.0714 & 0.0870 & 0.0932 \\
\hline$P_{2}$ & 0.0697 & 0.1190 & 0.1077 & 0.0401 & 0.1057 \\
\hline$P_{3}$ & 0.0771 & 0.1429 & 0.0796 & 0.0248 & 0.0777 \\
\hline$P_{4}$ & 0.0677 & 0.0687 & 0.0445 & 0.2017 & 0.0601 \\
\hline$P_{5}$ & 0.0603 & 0.1552 & 0.0294 & 0.1236 & 0.1003 \\
\hline
\end{tabular}

Next, the proposed modified TOPSIS is adopted for AirP-PSS scheme assessment to achieve scheme optimization.

According to $Y=\left[y_{i, t}\right]_{5 \times 5}$, the positive ideal solution is obtained as $Y^{+}=[0.1030,0.1552,0.1077$, $0.2017,0.1057]$ and the negative ideal solution is obtained as $Y^{-}=[0.0603,0.0494,0.0294,0.0248$, 0.0601].

Set pair $\left\langle Y_{1}, Y^{+}>\right.$consists of five element pairs $<y_{1,1}, y_{1}^{+}>,<y_{1,2}, y_{2}^{+}>, \ldots,<y_{1,5}, y_{5}^{+}>$. For element pair $\left\langle y_{1,3}, y_{3}^{+}\right\rangle$, the relational degree of $\left\langle y_{1,3}, y_{3}^{+}\right\rangle$ is obtained as $\rho_{1,3}^{+}=0 \cdot \Delta^{\prime}+0 \cdot \Delta^{\prime \prime}+0.5364 \cdot \Delta^{\prime \prime \prime}$. The relational degrees of other element pairs in $\left\langle Y_{1}, Y^{+}\right\rangle$ can be obtained similarly. Then, the relational vector of set pair $\left\langle Y_{1}, Y^{+}\right\rangle$is obtained as $\mu_{1}^{+}=[0.2000,0.2000,0.3229]$. In the same manner, the relational vector of set pair $\left\langle Y_{1}, Y^{>}>\right.$is obtained as $\mu_{1}^{-}=[0.2000,0.2000,0.2771]$.

The relational vector of the set pair $\left\langle Y_{1}, Y_{1}\right\rangle$ is $\mu_{1}^{1}=[1,0,0]$. Based on the relational vectors of $\left\langle Y_{1}, Y^{+}\right\rangle,\left\langle Y_{1}, Y^{>}\right\rangle$and $\left\langle Y_{1}, Y_{1}\right\rangle$, we obtain that the relational vector distances from $Y_{1}$ to $Y^{+}$and from $Y_{1}$ to $Y^{-}$are $\tau_{1}^{+}=0.8856$ and $\tau_{1}^{-}=0.8699$. Therefore, the closeness of $Y_{1}$ to the positive ideal solution is calculated as $\lambda_{1}=0.4955$.

Similarly, the closeness values of other assessment objects to the positive ideal solution are calculated as $\lambda_{2}=0.6463, \lambda_{3}=0.4371, \lambda_{4}=0.5356$ and $\lambda_{5}=0.4060$.
According to the arranging rule of TOPSIS, five AirPPSS schemes are arranged as $P_{2}>P_{4}>P_{1}>P_{3}>P_{5} . P_{2}$ is the best AirP-PSS scheme, and the scheme selection optimization is achieved.

\section{CONCLUSION}

Because the influencing factors of PSS scheme selection optimization have dynamic multidimensional characteristics and a complex coupling relationship in the optimization process, static assessment and selection optimization are with greater restrictions and unreasonable. Aiming at this problem, a digital twin driven framework to enhance PSS scheme selection optimization is presented, and its feasibility is verified by a case of air purification PSS scheme selection optimization. This paper explores the application of digital twin-driven frameworks in the optimization of PSS scheme selection. Although the work enhances PSS scheme selection optimization, some limitations call for future research. For example, the assessment data modelling of digital twin-enabled PSS scheme selection could be considered to provide a detailed information-analysing methodology. More data and cases should be used to improve the approach's feasibility and practicality.

\section{ACKNOWLEDGEMENTS}

This work is supported by the Curriculum Reform Project of Teacher Education in Henan Province (B054), Ningxia Natural Science Foundation (2020AAC03202) and the Third Batch of Ningxia Youth Talents Supporting Program (TGJC2018048). The authors also would thank Hongxia Sun, Bingbing Lei, Chuntao Zhang and Chunlei Mao for their contributions to this work.

\section{REFERENCES}

[1] Chiu, M.-C., Chu, C.-Y., Kuo, T.-C. (2019). Product service system transition method: building firm's core competence of enterprise. International Journal of Production Research, vol. 57, no. 20, p. 6452-6472, D0l:10.1080/00207543.2019.156 6670.

[2] Kim, S., Christiaans, H., Baek, J.-S. (2019). Smart Homes as product-service systems: Two focal areas or developing competitive smart home appliances. Service Science, vol. 11, no. 4, p. 292-310, D0l:10.1287/serv.2019.0248.

[3] Liu, Z., Ming, X. (2019). A framework with revised roughDEMATEL to capture and evaluate requirements for smart industrial product-service system of systems. International Journal of Production Research, vol. 57, no. 22, p. 7104-7122, DOI: 10.1080/00207543.2019.1577566. 
[4] Meier, H., Roy, R., Seliger, G. (2010). Industrial ProductService systems-IPS2. CIRP Annals, vol. 59, no. 2, p. 607-627, D0I:10.1016/j.cirp.2010.05.004.

[5] Sun, H., Wang, Z., Zhang, Y., Chang, Z., Mo, R., Liu, Y. (2012). Evaluation method of product-service performance. International Journal of Computer Integrated Manufacturing, vol. 25, no. 2, p. 150-157, Dol:10.1080/095119 2X.2011.627946.

[6] Yoon, B., Kim, S., Rhee, J. (2012). An evaluation method for designing a new product-service system. Expert Systems with Applications, vol. 39, no. 3, p. 3100-3108, D0l:10.1016/j. eswa.2011.08.173.

[7] An, X., Niu, C., Xue D., Mu, G., Lin, X. (2016). Optimal selection method for product service system scheme based on variable granule weight and group decision-making. Computer Integrated Manufacturing Systems, vol. 22, no. 1, p. 155-165, DOI: 10.13196/j.cims.2016.01.015. (in Chinese)

[8] Lan, S., Zhang, H., Zhong, R.Y., Huang, G.Q. (2016). A customer satisfaction evaluation model for logistics services using fuzzy analytic hierarchy process. Industrial Management \& Data Systems, vol. 116, no. 5, p. 1024-1042, D0l:10.1108/IMDS09-2015-0389.

[9] Chou, C.-J., Chen, C.-W., Conley, C. (2015). An approach to assessing sustainable product-service systems. Journal of Cleaner Production, vol. 86, p. 277-284, D0l:10.1016/j. jclepro.2014.08.059.

[10] Liu, Q., Zhang, H., Leng J., Chen X. (2019). Digital twindriven rapid individualised designing of automated flow-shop manufacturing system. International Journal of Production Research, vol. 57, no. 12, p. 3903-3919, D0l:10.1080/0020 7543.2018.1471243.

[11] Zhang, K., Qu, T., Zhou, D., Jiang, H., Lin, Y., Li, P., Guo, H., Liu, Y., Li, C., Huang G.Q. (2020). Digital twin-based optistate control method for a synchronized production operation system. Robotics and Computer-Integrated Manufacturing, vol. 63, p. 1-15, D0I:10.1016/j.rcim.2019.101892.

[12] Leng, J., Zhang, H., Yan, D., Liu, Q., Chen, X., Zhang, D. (2019). Digital twin-driven manufacturing cyber-physical system for parallel controlling of smart workshop. Journal of Ambient
Intelligence and Humanized Computing. vol. 10, p. 11551166, D0I:10.1007/s12652-018-0881-5.

[13] Li, L.-H., Hang, J.-C., Gao, Y., Mu, C.-Y. (2017). Using an integrated group decision method based on SVM, TFN-RSAHP, and TOPSIS-CD for cloud service supplier selection. Mathematical Problems in Engineering, vol. 2017, p. 1-15, DOI:10.1155/2017/3143502.

[14] Li, L.-H., Hang, J.-C., Sun, H.-X., Wang, L. (2017). A conjunctive multiple-criteria decision-making approach for cloud service supplier selection of manufacturing enterprise. Advances in Mechanical Engineering, vol. 9, no. 3, pp. 1-14, DOI:10.1177/1687814016686264.

[15] Geng, X., Xu S., Li Y., You, X. (2015). Product service system concept evaluating approach considering combinatorial effect between product and service. Computer Integrated Manufacturing Systems, vol. 21, no. 10, p. 2798-2806, DOl:10.13196/j.cims.2015.10.029. (in Chinese)

[16] Yuvaraj, T., Suresh, P. (2019). Analysis of EDM process parameters on inconel 718 using the grey-Taguchi and Topsis methods. Strojniški vestnik - Journal of Mechanical Engineering, vol. 65, no. 10, p. 557-564, Dol:10.5545/svjme.2019.6194.

[17] Alhabo, M., Zhang, L. (2018). Multi-Criteria Handover Using Modified Weighted TOPSIS Methods for Heterogeneous Networks. IEEE Access, vol. 6, p. 40547-40558, D0l:10.1109/ ACCESS.2018.2846045.

[18] Li, L.-H., Mao, C.-L., Sun, H.-X., Yuan, Y.-P., Lei, B.-B. (2020). Digital twin driven green performance evaluation methodology of intelligent manufacturing: Hybrid model based on fuzzy rough-sets AHP, multistage weight synthesis, and PROMETHEE II. complexity, vol 2020, p. 1-24, DOl:10.1155/2020/3853925.

[19] Yang, M., Zhang, L., Cui, Y., Zhou, Y., Chen, Y., Yan, G. (2020). Investigating the wind power smoothing effect using set pair analysis. IEEE Transactions on Sustainable Energy, vol. 11, no. 3, p. 1161-1172, D0I:10.1109/TSTE.2019.2920255.

[20] Garg, H., Kumar, K. (2020). A novel possibility measure to interval-valued intuitionistic fuzzy set using connection number of set pair analysis and its applications. Neural Computing \& Applications, vol. 32, no. 8, p. 3337-3348, DOl:10.1007/s00521-019-04291-w. 